AFRL-RD-PS-

AFRL-RD-PS-

TP-2013-0010

TP-2013-0010

\title{
METAMATERIAL CATHODES in MULTI-CAVITY MAGNETRONS
}

POSTPRINT

Andrey D. Adreev

Kyle J. Hendricks

28 April 2011

Technical Paper

APPROVED FOR PUBLIC RELEASE; DISTRIBUTION IS UNLIMITED.

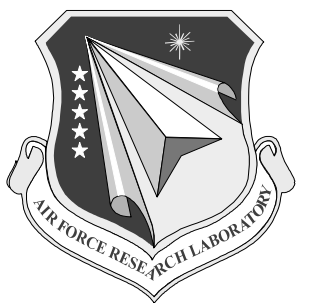

AIR FORCE RESEARCH LABORATORY Directed Energy Directorate 3550 Aberdeen Ave SE 


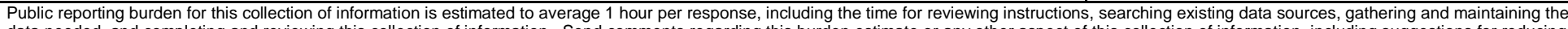

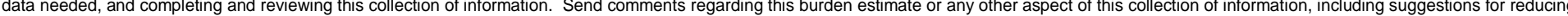

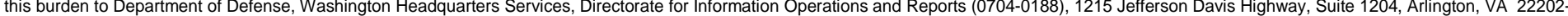

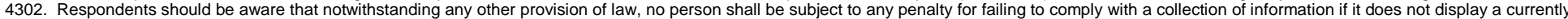
valid OMB control number. PLEASE DO NOT RETURN YOUR FORM TO THE ABOVE ADDRESS

\begin{tabular}{l|l|l|l} 
1. REPORT DATE (DD-MM-YYYY) & 2. REPORT TYPE & 3. DATES COVERED (FrOm - TO)
\end{tabular}

28 April $2011 \quad$ Technical Paper

\section{TITLE AND SUBTITLE}

METAMATERIAL CATHODES IN MULTI-CAVITY MAGNETRONS

(POSTPRINT)

Andrey D. Andreev, Kyle J. Hendricks

\section{8-2011 \\ 5a. CONTRACT NUMBER \\ CRADA}

5b. GRANT NUMBER

5c. PROGRAM ELEMENT NUMBER

5d. PROJECT NUMBER

5e. TASK NUMBER

5f. WORK UNIT NUMBER

7. PERFORMING ORGANIZATION NAME(S) AND ADDRESS(ES)

Air Force Research Laboratory

8. PERFORMING ORGANIZATION REPORT

3550 Aberdeen Avenue SE

Kirtland AFB, NM 87117-5776 NUMBER

\section{SPONSORING / MONITORING AGENCY NAME(S) AND ADDRESS(ES)}

Air Force Research Laboratory

3550 Aberdeen Avenue SE

Kirtland AFB, NM 87117-5776

10. SPONSOR/MONITOR'S ACRONYM(S)

AFRL/RDHP

11. SPONSOR/MONITOR'S REPORT NUMBER(S)

AFRL-RD-PS-TP-2013-0010

\section{DISTRIBUTION I AVAILABILITY STATEMENT}

Approved for public release; distribution is unlimited.

\section{SUPPLEMENTARY NOTES}

377ABW-2011-0600 Cleared on 04/22/2011

$18^{\text {th }}$ IEEE International Pulsed Power Conference

Presented on 06/19/2011

Government Purpose Rights

\section{ABSTRACT}

The paper presents results of computer experiments performed for research to enhance operational parameters of multi-cavity magnetrons through using the metal-thin-wire (MTW) and the metal-split-ring (MSR) metamaterial structures as a cold (nonthermionic) cathode. It is demonstrated by particle-in-cell (PIC) simulations of a generic 10-vane non-relativistic ultra-high frequency (UHF) magnetron that the output microwave power and the start-up time of this magnetron are improved by replacing either a solid or a transparent cathode by a bulk MTW metamaterial structure formed by an array of individual wires directed parallel to the axis of the magnetron (rodded cathode).

\section{SUBJECT TERMS}

Magnetron, metameterial, cathodes

\begin{tabular}{|c|c|c|c|c|c|}
\hline \multicolumn{3}{|c|}{ 16. SECURITY CLASSIFICATION OF: } & \multirow{2}{*}{$\begin{array}{l}\text { 17. LIMITATION } \\
\text { OF ABSTRACT } \\
\text { SAR }\end{array}$} & \multirow{2}{*}{$\begin{array}{c}\text { 18. NUMBER } \\
\text { OF PAGES } \\
9\end{array}$} & \multirow{2}{*}{$\begin{array}{l}\text { 19a. NAME OF RESPONSIBLE PERSON } \\
\text { Kyle Hendricks } \\
\text { 19b. TELEPHONE NUMBER (include area } \\
\text { code) } \\
\text { 505-853-3915 }\end{array}$} \\
\hline $\begin{array}{l}\text { a. REPORT } \\
\text { UNCLASS }\end{array}$ & $\begin{array}{l}\text { b. ABSTRACT } \\
\text { UNCLASS }\end{array}$ & $\begin{array}{l}\text { c. THIS PAGE } \\
\text { UNCLASS }\end{array}$ & & & \\
\hline
\end{tabular}




\section{METAMATERIAL CATHODES IN MULTI-CAVITY MAGNETRONS Andrey D. Andreev" and Kyle J. Hendricks High-Power Microwave Division, Directed Energy Directorate, Air Force Research Laboratory 3550 Aberdeen Ave, Kirtland Air Force Base, NM 87117-5776, USA}

Abstract

The paper presents results of computer experiments performed for research to enhance operational parameters of multicavity magnetrons through using the metal-thin-wire (MTW) and the metal-split-ring (MSR) metamaterial structures as a cold (non-thermionic) cathode. It is demonstrated by particle-in-cell (PIC) simulations of a generic 10-vane non-relativistic ultra-high frequency (UHF) magnetron that the output microwave power and the start-up time of this magnetron are improved by replacing either a solid or a transparent cathode by a bulk MTW metamaterial structure formed by an array of individual wires directed parallel to the axis of the magnetron (rodded cathode).

\section{INTRODUCTION}

The artificial MTW and the MSR structures possess metamaterial properties, i.e., negative- $\varepsilon /$ positive $-\mu$ and positive- $\varepsilon /$ negative- $\mu$, respectively, when the incident time-varying electric field $E_{1}$ oscillates along the wires of the MTW structure, or when the incident time-varying magnetic $H_{1}$ field oscillates perpendicularly to the rings of the MSR structure [1], [2]. In both cases, the incident $E_{1}$ and $H_{1}$ fields induce electric currents flowing along the individual metal wires (rings). The oscillations of the induced currents generate the artificial electric or magnetic dipole moments within the bulk of a metamaterial structure, which either constructively or destructively interact with the incident fields. Both MTW and MSR structures may be considered as quasi-metamaterials (transparent) structures when the incident time-varying electric field $E_{1}$ is orthogonal to the individual wires and freely penetrates inside of those structures.

The multi-cavity magnetron operates in one of the transverse-electrical-like (TE-like) cavity modes of the magnetron resonant cavity (magnetron diode) formed by the cathode emitting electrons and the anode consisting of a number of resonators [3], [4]. The resonant cavity slows down the induced electromagnetic wave azimuthally traveling between the cathode and the anode. The spatial distribution of the time-varying electromagnetic field $\left(E_{1} \times H_{1}\right)$, induced within the resonant cavity and associated with the traveling electromagnetic wave, is characterized by the circumferential $E_{1 \phi}$ and the radial $E_{1 \rho}$ components of the electric field and the axial $H_{1 z}$ component of the magnetic field. The number of $E_{1 \phi}$ phase variations in the azimuthal direction is determined by the operating frequency of the magnetron (mode of the magnetron operation) and the anode geometry (number of cavities). The number of $E_{1 \rho}$ and $H_{1 \mathrm{z}}$ phase variation along the radius and the axis of the resonant system are usually minimized

\footnotetext{
"Andrey D. Andreev is an NRC Associate at the AFRL's Directed Energy Directorate.
}

to one. This allows the magnetron to operate in the lowerfrequency $T E$-like mode (at the given number of $E_{1 \phi}$ circumferential variations) and prevents axial and radial mode competition. The external electric field $E_{0}$ in the magnetron diode is directed radially from the anode to the cathode. The external magnetic field $H_{0}$ is directed axially. The crossed $E_{0} \times H_{0}$ fields force electrons emitted from the cathode to drift in the azimuthal direction. The multicavity magnetron starts to generate (induce) $E_{1} \times H_{1}$ fields and produce microwaves (output power) when the drift velocity of the emitted electron is approximately equal to the phase velocity of $E_{1 \phi}$.

For the multi-cavity magnetron operating in the TE-like mode, the bulk metamaterial cathode may be designed in the form of a single/double/multi-helix structure (Fig. 1a), the individual elements (turns) of which are oriented parallel to $E_{1 \phi}$ and perpendicular to $H_{1 \mathrm{z}}$. The proper choosing of the helix pitch may allow implementing the negative- $\varepsilon /$ negative- $\mu$ properties of the metamaterial cathode within the desired microwave frequency range to enhance and improve the magnetron operational parameters. The metamaterial cathode may be also designed in the form of the rodded medium whose individual elements (rods) are placed either radially (Fig. 1b) (parallel to $E_{1 \rho}$ and perpendicular to both $E_{1 \phi}$ and $H_{1 \mathrm{z}}$ ) of longitudinally (Fig. 1c) (perpendicular to both $E_{1 \rho}$ and $E_{1 \phi}$ and parallel to $H_{1 z}$ ). In the latter case (Fig. 1c), the rodded medium is essentially a quasi-metamaterial structure.
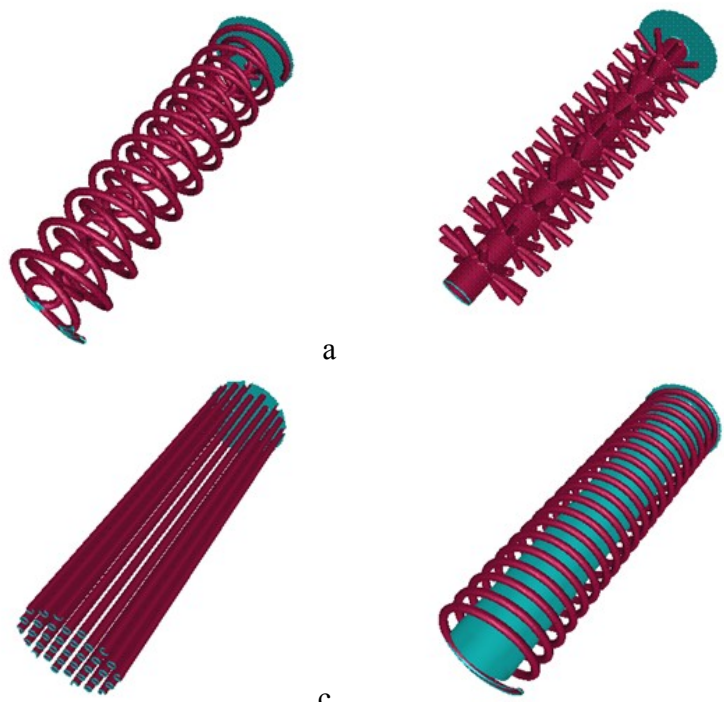

a

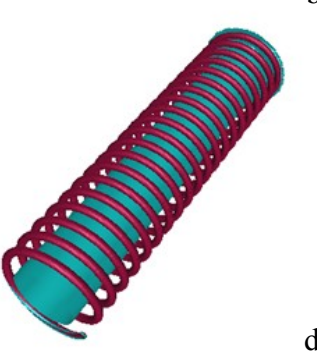

b d

Fig. 1. The different cathodes: a) multi-helix structure with metal wires parallel to $E_{1 \phi}$ and the metal rings perpendicular to $H_{1 \mathrm{z}}$, b) multi-point structure with metal wires parallel to $E_{1 \rho}$ (multipoint cathode), c) multi-rod structure with metal wires parallel to $H_{1 z}$ (rodded cathode), d) standard helical cathode with nonemitting central rod. 
Results of the PIC simulations of a generic 10-vane non-relativistic continuous-wave (CW) UHF magnetron with a quasi-metamaterial multi-rod or rodded cathode (Fig. 1c) are described below. Most important geometrical dimensions of the magnetron are similar to the commercially available CWM-75/100L and L-4031 tubes of the L-3 Communications, Electron Devices [5].

\section{SIMULATION GEOMETRY AND PROCEDURE}

The simulations are performed using ICEPIC code developed and maintained by the $A F R L / R D H E$ [6]-[8]. ICEPIC is a fully relativistic, three-dimensional (3D), variable-mesh PIC code capable of simulating the interdisciplinary physics of charge-particle beams, high-power microwaves (HPM), and plasmas in complex geometries of modern microwave vacuum electronic devices (MVEDs) [8]. The code incorporates numerous advances in parallel computing and performs automatic partitioning using a single domain decomposition algorithm, asynchronous message passing, and dynamic load balancing [7]. ICEPIC simulates from the first principles (Maxwell's equations and Lorentz's forces law) the relativistic electrodynamics and charged-particle dynamics within the resonant structures of different MVEDs [6].

The simulation model of the magnetron is built within the Cartesian $x-y-z$ geometry using simulation cells with grid size of $0.5 \times 0.5 \times 0.5 \mathrm{~mm}^{3}$ [9]. It consists of: (i) the cathode block with the input port, (ii) the 10-vane anode block surrounding the emitting part of the cathode block, (iii) the "double-ring-strapping" system coupling the alternative resonators of the anode block, and (iv) three output electrodes connecting three vanes of the anode block with the appropriate output ports.

The anode block consists of ten vanes, $N=10$, forming a cylindrical slow-wave structure (SWS). There are two grooves, one on each end of the anode block. The doublering-strapping system is located within the grooves on each end of the anode block. Each strap electrically connects alternate vanes and passes over adjacent vanes of the anode block. Three cylindrical electrodes connect the first, the fourth, and the sixth vanes of the anode block with the output ports. The cathode block consists of the non-emitting central electrode connecting the input port with the emitting part of the cathode, two non-emitting end caps preventing emitted electrons from leaking out the interaction space, and the cathode itself (Fig. 2).

The simulations of the magnetron are performed at input voltage $V_{\text {in }}=45 \mathrm{kV}$ and magnetic flux density $B_{0}=0.49$ $\mathrm{T}$. The rise time of $V_{\text {in }}$ is $10 \mathrm{~ns}$ [10] at simulation time of 2000 ns. This allows one to consider $V_{\text {in }}$ as the "steadystate" input parameter. $B_{0}$ is axially directed, uniform, and constant within the simulation volume and during the simulation time.

In the course of the simulations, $V_{\text {in }}$ is monitored (as well as the input current $I_{\text {in }}$ and the input power $P_{\text {in }}$ ) at the input port. The output power $P_{\text {out }}$ is monitored (as well as the output $r f$ voltage $V_{\text {out }}$ ) at the output ports. The resona- tor voltage $V_{\text {res }}$ is monitored inside the first resonator of the anode block. The anode current $I_{\mathrm{a}}$ is monitored at the plane located on a small distance (one or two simulation cells) from the anode surface. All monitored parameters are smoothed over 100,000 consecutive time steps and averaged over their steady-state phases (when the monitored parameter is not changed in time) in order to get the steady-state characteristics of the magnetron operation.

The entire simulation procedure develops as follows. Once $V_{\text {in }}$ is applied to the input port, the space-charge limited (SCL) algorithm of the ICEPIC code [11] begins to produce electrons at a small distance from the cathode (one or two grid cells). These originated electrons are extracted from the cathode and accelerated by $E_{0}$, deflected by $H_{0}=\mu_{0} B_{0}$ ( $\mu_{0}$ is permeability of vacuum), and move within the interaction space in accordance with the given distributions of $E_{0} \times H_{0}$ and the electron spacecharge $q_{\mathrm{e}}$ accumulated within the interaction space.

The accelerated electrons give rise to $I_{\text {in }}$ flowing along the central electrode connecting the input port with the cathode. After some amount of time, determined by the loaded $Q$ (quality factor [4]) of the magnetron resonant cavity, the magnetron spokes begin to grow and the magnetron starts to operate. After formation of the magnetron spokes, both $I_{\mathrm{a}}$ and $P_{\text {out }}$ increase and go into their steady-state phases. The electrons begin to move within the interaction space in accordance with the given distributions of $E_{0} \times H_{0}, E_{1} \times H_{1}$, and $q_{\mathrm{e}}$.

Results of the simulations of the magnetron having the rodded cathode (Fig. 1c, Fig. 2c) are compared with the results obtained during PIC simulations of the same magnetron having the solid cylindrical (Fig. 2a) and the transparent [12], [13] (Fig. 2b) cathodes. All cathodes of the simulation model of the magnetron (Fig. 2) are supposed to operate in the explosive electron emission (EEE) mode [14], which automatically assumes the space-charge limited (SCL) mechanism of the electron extraction from the cathode into the interaction space. Note, however, that the standard high-power industrial-heating UHF CWM75/100L and L-4031 MHz magnetrons have the helical thermionic cathode (with the central back-current electrode) (Fig. 1d) working in the thermal electron-emission (TEE) mode [5].

\section{SIMULATION RESULTS}

The particle plot distributions within the interaction space of the magnetron having three different cathodes (Fig. 2) (solid, transparent, and rodded) are shown in Fig. 3 ( $\sim 10 \mathrm{~ns}$ of simulation time), Fig. 4 ( $\sim 100 \mathrm{~ns})$, and Fig. 5 ( $\sim 1000 \mathrm{~ns})$. It can be seen in Fig. 3-Fig. 5 that the magnetron begins to oscillate in the $\pi$-mode with either cathode. The $\pi$-mode is recognized by five magnetron spokes corresponding to the $T E_{51}$ mode of $E_{1} \times H_{1}$ distribution within the interaction space.

There are, however, very significant differences in the amount of the anode current $I_{\mathrm{a}}$ and the output power $P_{\text {out }}$ when the magnetron operates with the different cathodes. 

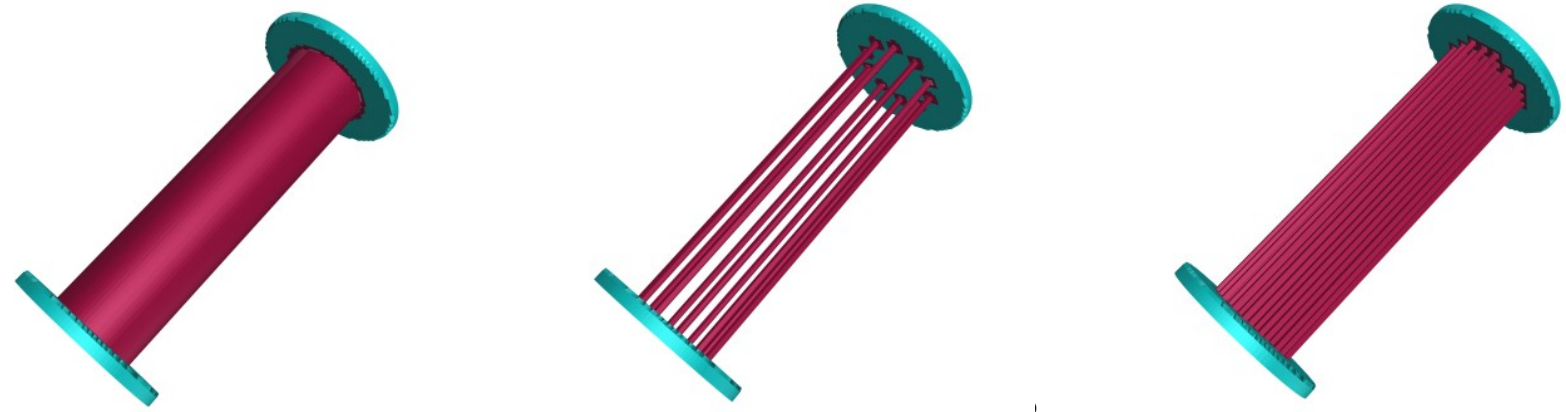

Fig. 2. Cathodes used in simulations: a) solid cathode, b) transparent cathode, c) rodded (quasi-metamaterial) cathode (Fig. 1c).

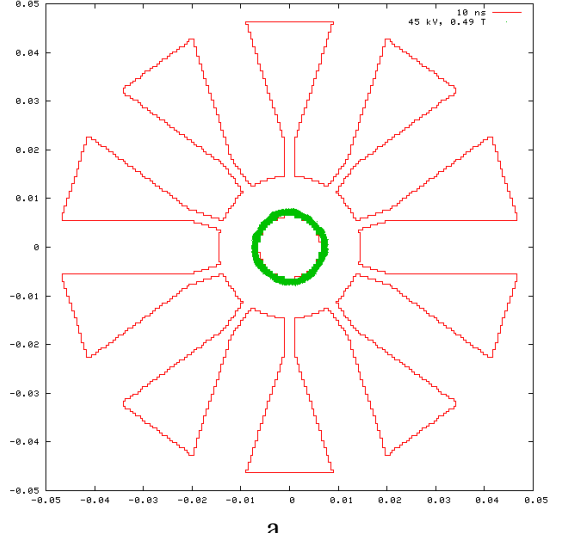

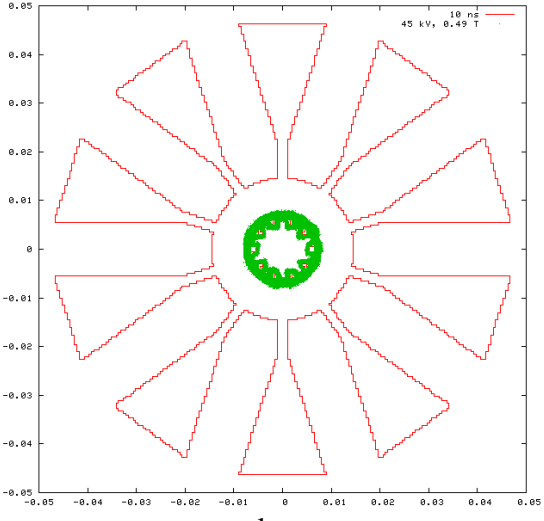

$\mathrm{b}$

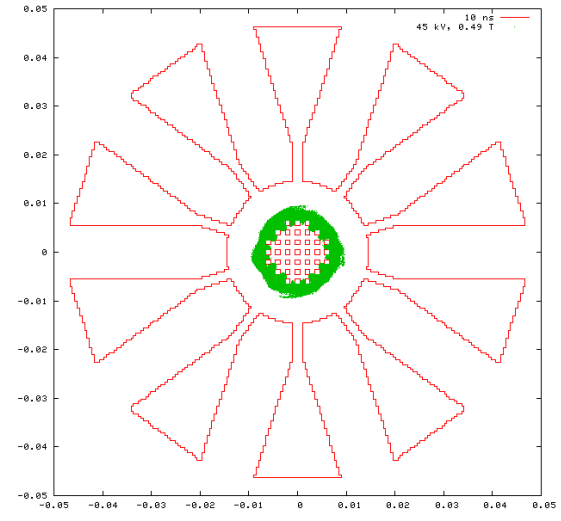

$\mathrm{c}$

Fig. 3. Particle plots at $10 \mathrm{~ns}$ of simulation time: a) solid cathode, b) transparent cathode, c) rodded (quasi-metamaterial) cathode.
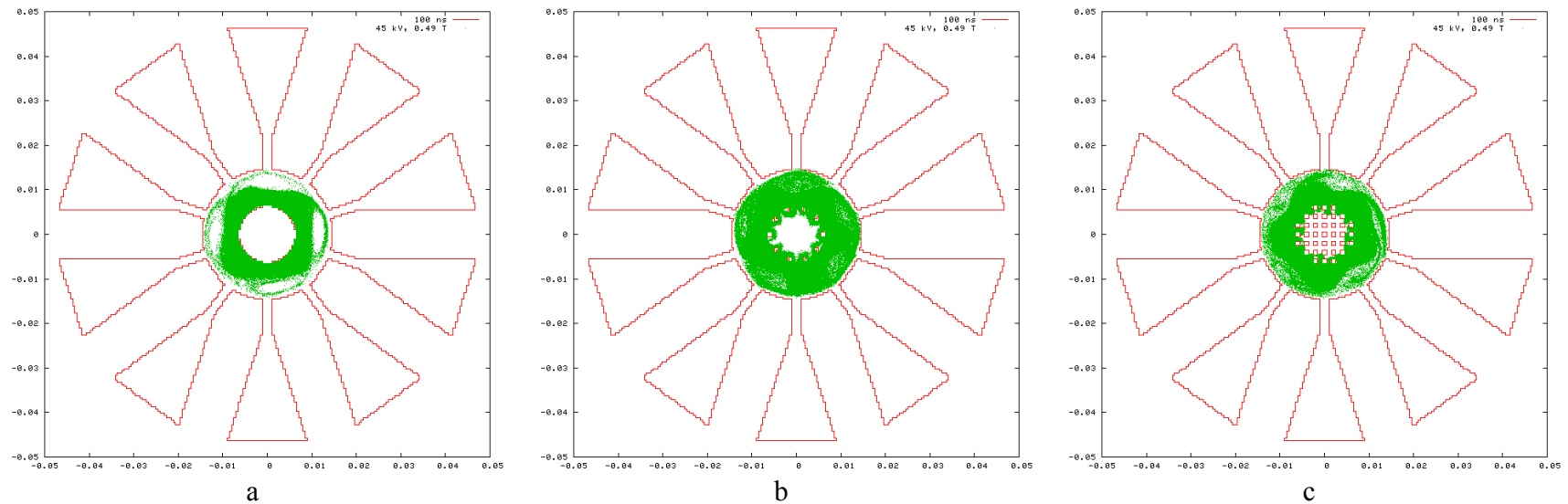

Fig. 4. Particle plots at 100 ns of simulation time: a) solid cathode, b) transparent cathode, c) rodded (quasi-metamaterial) cathode.

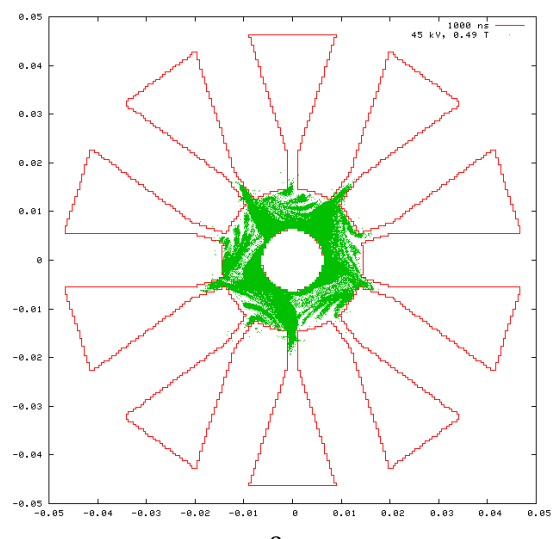

a

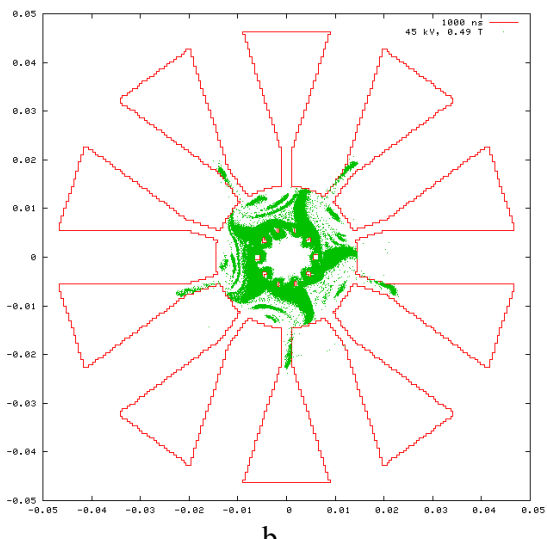

b

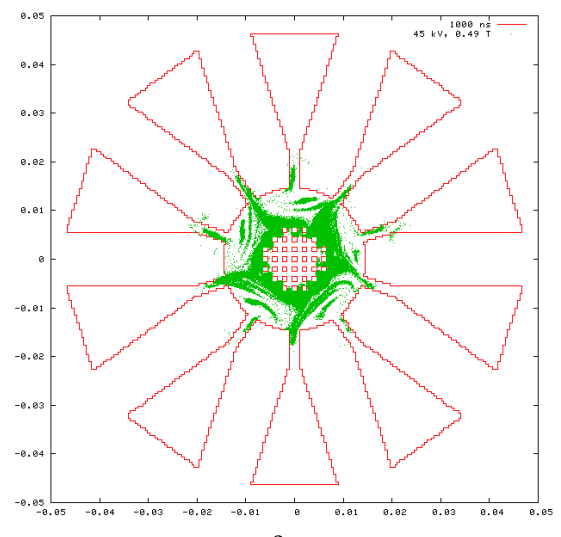

$\mathrm{c}$

Fig. 5. Particle plots at 1000 ns of simulation time: a) solid cathode, b) transparent cathode, c) rodded (quasi-metamaterial) cathode. 


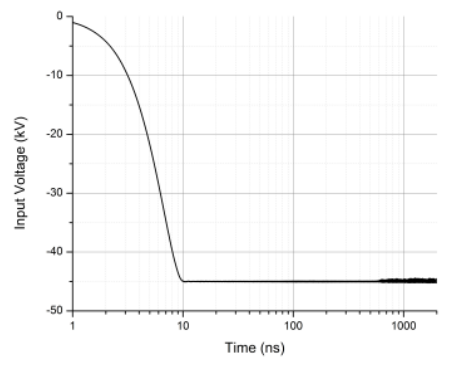

$\mathrm{a}$

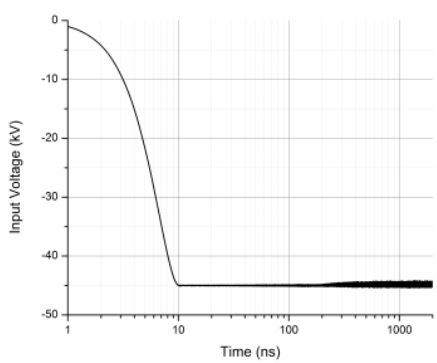

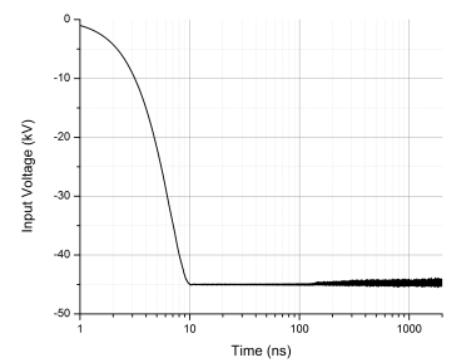

b

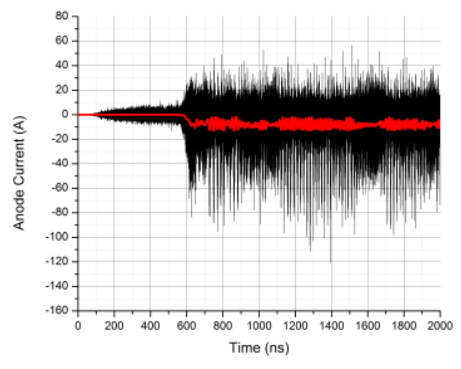

a

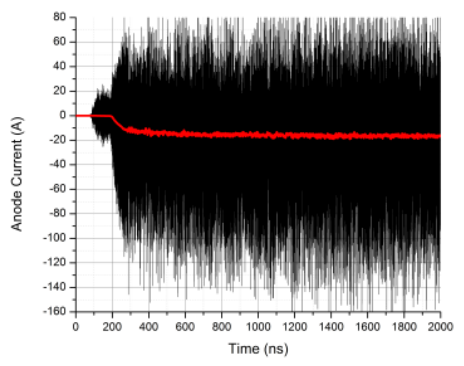

b

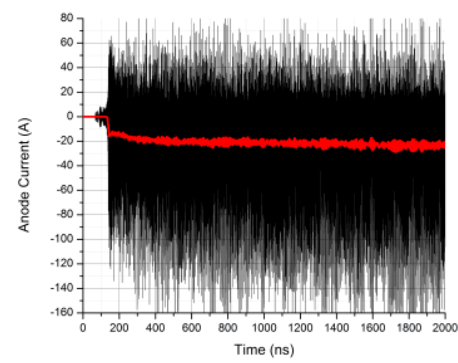

Fig. 7. Anode Current, $I_{\mathrm{a}}$ : a) solid cathode, b) transparent cathode, c) rodded (quasi-metamaterial) cathode.
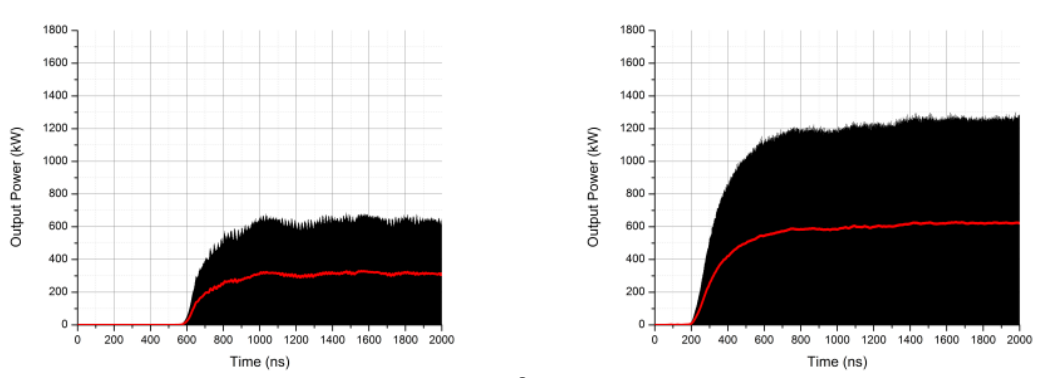

a

Fig. 8. Output Power, $P_{\text {out }}$

b

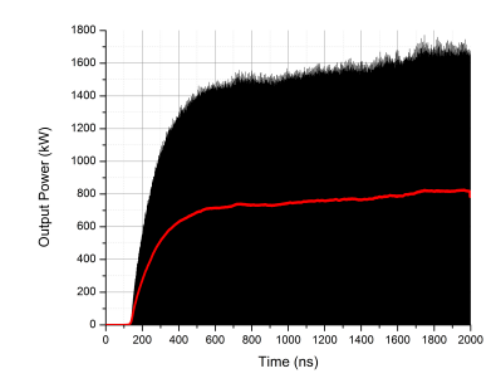

b

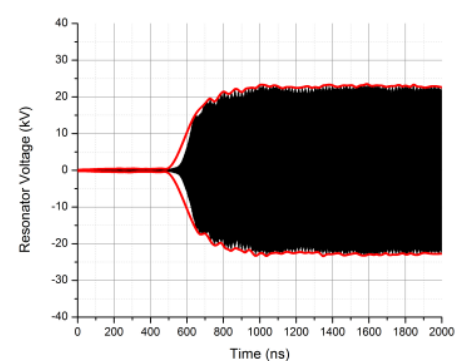

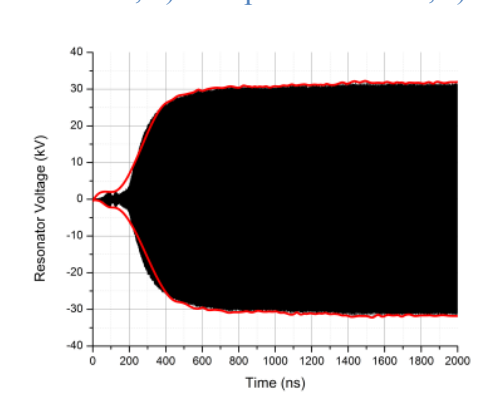

a

Fig. 9. Resonator Voltage, $V_{\text {res }}$ : a)
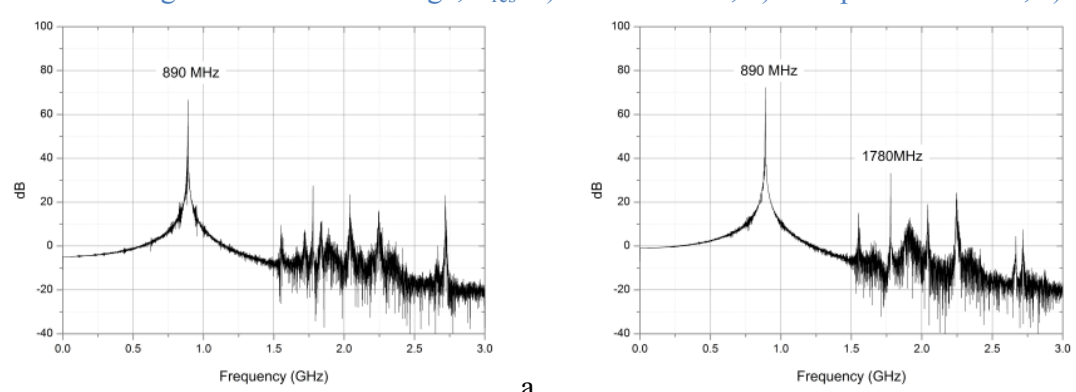

b

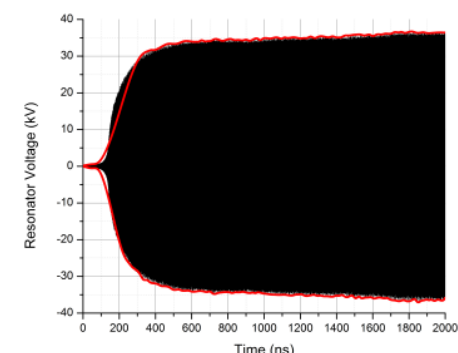

b

quasi-metamaterial) cathode.

Fig. 10. Spectrum of Output Voltage, $V_{\text {out }}$ a) solid cathode, b) transparent cathode, c) rodded (quasi-metamaterial) cathode. 
The differences can be seen in Fig. 7 that the steadystate $I_{\mathrm{a}}$ increases from $\sim 7.7 \mathrm{~A}$ in the magnetron having solid cathode, to $\sim 16.7 \mathrm{~A}$ in the magnetron having transparent cathode, and up to $\sim 23.1 \mathrm{~A}$ in the magnetron having rodded cathode. In the exact accordance with $I_{\mathrm{a}}$ increase [15], [16], the steady-state $P_{\text {out }}$ increases from $\sim 311$ $\mathrm{kW}$ in the magnetron having solid cathode, to $\sim 621 \mathrm{~kW}$ in the magnetron having transparent cathode, and up to $\sim 820$ $\mathrm{kW}$ in the magnetron having rodded cathode (Fig. 8).

Detailed analysis of the obtained results show that both $I_{\mathrm{a}}$ (Fig. 7) and $P_{\text {out }}$ (Fig. 8) increase in direct proportion to the square of the resonator voltage $V_{\text {res }}$ (Fig. 9), which is directly proportional to the induced time-varying electric field amplitude $E_{1}$. The frequency of the magnetron operation $f_{\pi}$ is determined by the frequency of $E_{1}$ oscillations. Frequency $f_{\pi}$ remains the same with all cathodes (Fig. 10).

The numerical relations between $I_{\mathrm{a}}, P_{\text {out }}$ and $V_{\text {res }}$ demonstrate that both $I_{\mathrm{a}}$ and $P_{\text {out }}$ increase in direct proportion to the square of $V_{\text {res. }}$. This allows one to suppose that the direct cause of both $I_{\mathrm{a}}$ and $P_{\text {out }}$ increase is nothing but the $E_{1}$ increase within the interaction space. The smoothed traces of $I_{\mathrm{a}}$ and $P_{\text {out }}$ are shown in Fig. 11 and Fig. 12, correspondently. The envelope of $V_{\text {res }}$ is shown in Fig. 13. All traces demonstrate how the cathode construction (Fig. 2) affects the magnetron operation.

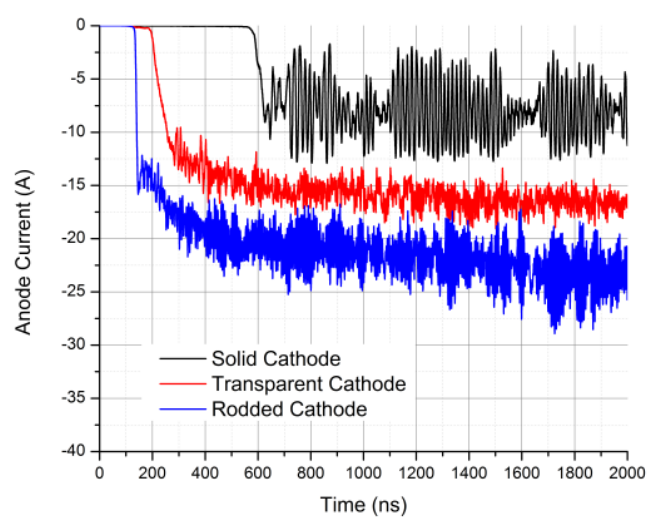

Fig. 11. Smoothed traces of the anode current $I_{\mathrm{a}}$.

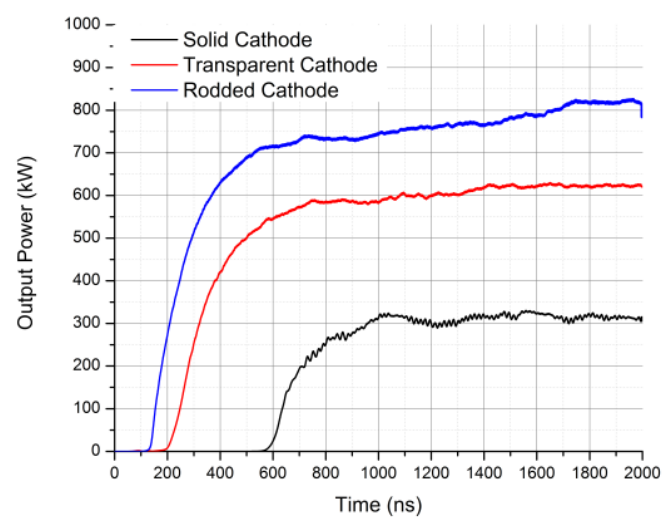

Fig. 12. Smoothed traces of the output power $P_{\text {out }}$.

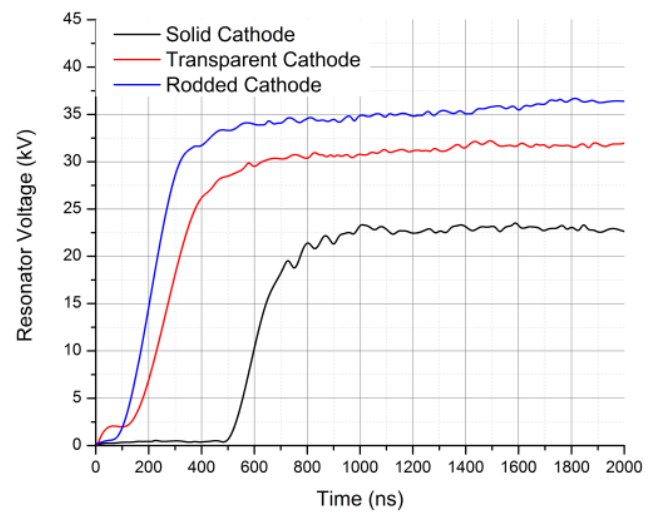

Fig. 13. Envelope of the resonator voltage $V_{\text {res }}$.

\section{CONCLUSION}

PIC simulations of a generic 10-vane UHF magnetron with the solid, the transparent, and the quasi-metamaterial (rodded) cathodes (Fig. 2) are performed. Results of the simulation showed that the use of the rodded cathode allows significantly increasing the output microwave power (Fig. 12) by the appropriate increase of the anode current (Fig. 11) of the magnetron. The anode current increase is caused by appropriate increase of the time-varying induced electric field $E_{1}$ (square of the resonator voltage (Fig. 13)) within the interaction space. The latter effect is the direct result of the metamaterial structure (rodded cathode) use within the magnetron resonant cavity.

\section{REFERENCES}

[1] L. Solimar and E. Shamonina, Waves in Metamaterial, Oxford University Press, 2009.

[2] C. Caloz and T. Itoh, Electromagnetic Metamaterials: Transmission Line Theory and Microwave Applications, John Wiley \& Sons, Inc., 2006.

[3] R.M. Gilgenbach, Y.Y. Lau, H. McDowell, K.L. Cartwright, and T.A. Spencer, "Crossed-field devices," in Modern Microwave and Millimeter-Wave Power Electronics Edited by R.J. Barker, J.H. Booske, N.C. Luhmann, Jr, and G.S. Nusinovich, IEEE Press and John Wiley \& Sons, Inc., 2005, pp.289-342.

[4] J. Benford, J.A. Swegle, and E. Schamiloglu, "Relativistic magnetrons and MILOs," in High-Power Microwaves, Second Edition, CRC Press and Taylor \& Francis Group, 2007, pp.259-320.

[5] Wynn, A.P., D.E. Blank, P.S. Campbell, R.R. Lentz, W.T. Main, S.G. Tantawi, K.G. Kato, H.K. Beutel, K.W. Brown, D.D. Crouch, G.K. Jones, and R.B. McDonald, "Development of a $300 \mathrm{~kW} \mathrm{CW} \mathrm{L-band} \mathrm{industrial} \mathrm{heating} \mathrm{magne-}$ tron," in Fifth IEEE International Vacuum Electronics Conference IVEC 2004, April 27-29, 2004, pp.164-165.

[6] G.E. Sasser, L. Bowers, S. Colella, D. Lileikis, J. Luginsland, D. McGrath, L. Merkle, R.E. Peterkin, and J.J. Watrous, "Virtual prototyping of microwave devices using MHD, PIC, and CEM codes," Proceedings of the $29^{\text {th }}$ AIAA Plasmadynamics and Lasers Conference, Albuquerque, NM, USA, 15-18 June 1998, publication \# AIAA-98-2926, AIAA Press, 1998. 
[7] K.L. Cartwright, A.G. Greenwood, P.J. Mardahl, T. Murphy, and M.D. Haworth, "Virtual prototyping of radio frequency weapons," Journal of Directed Energy, vol. 1, no. 1, Fall 2003, pp. 16-34.

[8] D. Shiffler, T.K. Staton, T.W. Hussey, O. Zhou, and P. Mardahl, "High-power microwave sources," in Modern Microwave and Millimeter-Wave Power Electronics, edited by R.J. Barker, J.H. Booske, N.C. Luhmann Jr., and G.S. Nusinivich, John Wiley \& Sons, 2005, pp.691-730.

[9] A.D. Greenwood, "An ICEPIC convergence study using a relativistic magnetron," Air Force Research Laboratory Technical Memorandum, AFRL-DE-PS-TM-2005-1005, 2005.

[10] D. Price, E. Goldman, D. Nett, S. Putnam, D. Weidenheimer, C. Bloemker, R. White, and T. Wynn, "DEW technology at the Titan Corporation," Proceedings of the $1^{\text {st }}$ AIAA International Energy Conversion Engineering Conference, Portsmouth, Virginia, USA, 17-21 August 2003, publication \# AIAA-2003-5913, AIAA Press, 2003.

[11] J.J. Watrous, J.W. Luginsland, and G.E. Sasser III, “An improved space-charge-limited emission algorithm for use in particle-in-cell codes," Physics of Plasmas, vol. 8, no. 1, pp. 289-296, January 2001.

[12] M. Fuks and E. Schamiloglu, "Rapid start of oscillations in a magnetron with a "transparent" cathode," Physical Review Letters, vol. 95, no. 20, 11 November 2005, pp. 205101(1-4).

[13] M.C. Jones, V.B. Neculaes, Y.Y. Lau, R.M. Gilgenbach, W.M. White, B.W. Hoff, and N.M. Jordan, "Magnetron priming by multiple cathodes," Applied Physics Letters, vol. 87, 2005, pp. 081501(1-3).

[14] G.A. Mesyats, Explosive Electron Emission, URO Press, 1998.

[15] R.B. Miller, "The relativistic microwave magnetron," in $A n$ Introduction to the Physics of Intense Charged Particle Beams, Plenum Press, 1982, pp.214-236.

[16] A.D. Andreev, K.J. Hendricks, M.I. Fuks and E. Schamiloglu, "Elemental theory of a relativistic magnetron operation: Anode current, Journal of Directed Energy, vol. 3, no. 4, Summer of 2010, pp.349-383. 


\section{DISTRIBUTION LIST}

\section{DTIC/OCP}

8725 John J. Kingman Rd, Suite 0944

Ft Belvoir, VA 22060-6218

$1 \mathrm{cy}$

AFRL/RVIL

Kirtland AFB, NM 87117-5776

2 cys

Official Record Copy

AFRL/RDHP/Kyle Hendricks 1 cy 